\title{
Em busca do melhor aproveitamento de bolsas de concentrado de hemácia de baixo volume
}

\author{
In search of the best use of bags low-volume red blood cells concentrate
}

Adriana Guimarães Estácio ${ }^{1}$ (D), Lacy Cardoso de Brito Júnior ${ }^{2}$ (D)

\section{RESUMO}

Introdução: A produção de bolsas de sangue total com volume inferior ao esperado é prevista na Portaria de Consolidação no 05/2017, porém, a sua dispensação hoje leva em consideração apenas o teor de hemoglobinas e hematócrito da bolsa. Objetivo: Determinar, a partir dos parâmetros técnicos brasileiros existentes, os critérios para o melhor aproveitamento para bolsas de concentrados de hemácias de baixo volume (CHBV) produzidas na Fundação HEMOPA. Material e métodos: Foram analisados volume, teor de hemoglobina e hematócrito de 525 bolsas de $\mathrm{CHBV}$ provenientes de bolsas duplas sem adição de conservante; triplas convencional com adição de SAG-M (soro fisiológico, adenina, glicose e manitol); triplas top and bottom (TAB) com adição de SAG-M; e quádruplas TAB com filtro "in line" e adição de SAG-M. Resultados: Foi observado que 71,43\% (375/525) das bolsas de CHBV, independente do tipo da bolsa, apresentavam-se em conformidade para hematócrito e teor de hemoglobina em relação à legislação. As bolsas duplas e triplas convencionais apresentaram os maiores valores de teor de hemoglobina. Observou-se ainda que as bolsas de sangue total coletadas com volumes entre 350 e 404 $\mathrm{ml}$ geraram bolsas de CHBV com os menores índices de descarte. O maior descarte das bolsas de CHBV ocorreu quando o volume final $\leq 250 \mathrm{~mL}$ e hemoglobina $\leq 16 \mathrm{~g} / \mathrm{dL}$. Conclusão: Observou-se que o melhor aproveitamento das bolsas de CHBV ocorreu quando as bolsas de sangue total se encontravam com volumes entre 350 e $404 \mathrm{~mL}$ e as bolsas de CHBV apresentavam volume final $\geq 250 \mathrm{~mL}$ e hemoglobina $\geq 16 \mathrm{~g} / \mathrm{dL}$.

Palavras-chave: Transfusão sanguínea, Segurança transfusional, Concentrado de hemácias, Parâmetros, Segurança do paciente.

\begin{abstract}
Introduction: The production of whole blood bags with a lower volume than expected is approved in the Consolidation Ordinance number 05/2017; however, its dispensation today takes into account only the hemoglobin and hematocrit content of the bag. Objective: From the existing Brazilian technical parameters, determine the criteria for the best clinical use for low-volume red cell concentrate (LVRCC) bags produced at the HEMOPA Foundation. Material and methods: We analyzed volume, hemoglobin content, and hematocrit of 525 LVRCC bags from double bags without addition of preservative; conventional triple bags with the addition of SAG-M (saline, adenine, glucose, and mannitol); triples bags top and bottom (TAB) with the addition of SAG-M, and quadruple bags TAB with "in line" filter and the addition of SAG-M. Results: It was observed that $71.43 \%$ (375/525) of bags of LVRCC, regardless of bag type, were in conformance with hematocrit and hemoglobin content concerning Brazilian legislation. With the double and conventional triple bags, they had the highest values of hemoglobin content. It was also observed that the bags of whole blood collected with volumes between 350 and $404 \mathrm{~mL}$ generated LVRCC bags with the lowest rate of discard. The greatest discard of LVRCC bags occurred when the final volume $\leq 250 \mathrm{~mL}$ and hemoglobin $\leq$ $16 \mathrm{~g} / \mathrm{dL}$. Conclusion: It was observed that the best use of LVRCC bags occurred when the whole blood bags were between 350 and $404 \mathrm{~mL}$ and the LVRCC bags had a final volume $\geq 250 \mathrm{~mL}$ and hemoglobin $\geq 16 \mathrm{~g} / \mathrm{dL}$.
\end{abstract}

Keywords: Blood transfusion, Transfusion safety; Red cells concentrate, Parameters, Patient safety.

1. Universidade Federal do Pará (UFPA). Fundação Centro de Hemoterapia e Hematologia do Pará, Belém, (PA), Brasil

2. Universidade Federal do Pará. Instituto de Ciências Biológicas. Laboratório de Patologia Geral - Imunopatologia e Citologia, Belém, (PA), Brasil 


\section{INTRODUÇÃO}

Um dos maiores problemas enfrentados pelos hemocentros em todo o Brasil ainda é a baixa sensibilização da população para a doação de sangue e, por conseguinte, a manutenção dos estoques de sangue $e^{1,2,3}$. Isto porque não basta tão somente captar doadores, é necessário que esse candidato seja considerado apto à doação após triagem clínica; que a bolsa coletada tenha volume final entre 450 $\pm 45 \mathrm{~mL}$ de sangue; e ainda que os produtos derivados dessa bolsa de sangue (hemocomponentes) sejam considerados "conformes" para uso em uma transfusão após a realização de diversos testes hematológicos, sorológicos e de imunohematologia $4,5,6,7,8$.

No tocante especificamente em relação ao volume final da bolsa de sangue total coletada em cada doação de sangue, a Portaria de Consolidação no 05/2017 do Ministério da Saúde ${ }^{8}$ estabelece que bolsas de sangue total coletadas com volumes entre 300 e $404 \mathrm{~mL}$, embora não sejam desejáveis, são consideradas "conformes" para a produção de bolsas de $\mathrm{CH}$ apenas e desde que: a mesma receba etiqueta própria que a caracterize como uma bolsa de $\mathrm{CH}$ derivada de uma bolsa de sangue total de baixo volume; e essa esteja com os parâmetros de teor de hemoglobina (> 45g/und), hematócrito (50 a $80 \%$ ) e grau de hemólise ( $<0,8 \%$ da massa eritrocitária) dentro das margens de segurança aceitáveis ${ }^{3,7,7,8,910,11,12,13}$.

Neste sentido, em 2012, a Fundação HEMOPA através da gerência de controle de qualidade de hemocomponentes do Hemocentro Coordenador, visando reduzir o descarte de bolsas de concentrados de hemácias produzidas a partir de bolsas de sangue total de baixo volume, e consequentemente melhora do aproveitamento dessas bolsas, validou que para uma bolsa de $\mathrm{CH}$ ser incorporada aos estoques da fundação, o volume mínimo da bolsa de coleta de sangue total deveria ser igual ou superior a $350 \mathrm{~mL}$ independente do tipo de bolsa em que o sangue foi coletado (dados não apresentados).

Assim, o objetivo deste estudo foi determinar, a partir dos parâmetros técnicos brasileiros existentes, os critérios para o melhor aproveitamento para bolsas de concentrados de hemácias de baixo volume (CHBV) produzidos na Fundação HEMOPA.

\section{MATERIAL E MÉTODOS}

\section{Casuística}

Estudo retrospectivo, quantitativo e analítico para a obtenção de dados de teor de hemoglobina (g/und) e hematócrito (\%) de 525 bolsas de concentrado de hemácia de baixo volume (CHBV), provenientes da rotina da gerência de controle de qualidade de hemocomponentes (GECOQ) e que foram cadastradas no Sistema de Banco de Sangue (SBS) da unidade coordenadora da Fundação HEMOPA, no período de janeiro de 2015 a dezembro de 2018.

Para tanto, foram incluídas nesse estudo apenas os dados de teor de hemoglobina ( $\mathrm{g} / \mathrm{und}$ ) e hematócrito (\%) de bolsas de CHBV produzidas a partir de bolsas de sangue total coletadas com volumes entre 350 e $404 \mathrm{~mL}$. Os valores de hemoglobina e hematócrito dessas bolsas foram obtidos a partir do processamento de $3 \mathrm{~mL}$ de amostras de sangue total do espaguete dessas bolsas em contador hematológico automatizado da marca Sysmex, modelo KX 21N (Roche do Brasil). E o cálculo do teor de hemoglobina da bolsa de CHBV ( $\mathrm{g} / \mathrm{und}$ ) foi obtido através da fórmula: Teor de hemoglobina $(\mathrm{g} / \mathrm{und})=$ hemoglobina da bolsa de CHBV $(\mathrm{g} / \mathrm{dL})$ X volume da bolsa de CHBV $(\mathrm{mL}) / 100$, onde o volume das bolsas de CHBV (registrado no SBS) foi obtido através da fórmula: Volume = peso da bolsa de sangue total - tara da balança (peso da bolsa vazia)/Densidade do $\mathrm{CH}$ (aproximadamente 1,065 ).

\section{Descrição dos dados}

Os dados de teor de hemoglobina ( $\mathrm{g} / \mathrm{und}$ ) e hematócrito (\%) das bolsas de CHBV analisadas neste estudo foram separados em grupos conforme o tipo de bolsa de coleta de sangue total (Fresenius-Kabi®) em: Grupo $1(n=194)$, formado por dados provenientes de bolsas duplas sem adição de conservante; Grupo 2 $(n=110)$, formado por dados provenientes de bolsas triplas convencional com adição de soro fisiológico, adenina, glicose e manitol (SAG-M); Grupo $3(n=194)$, formado por dados provenientes de bolsas triplas top and bottom (TAB) com adição de SAG-M; e Grupo 4 $(n=27)$, formado por dados provenientes de bolsas quádruplas TAB com filtro "in line" e adição de SAG-M.

Tendo sido consideradas "conformes" as bolsas de CHBV que apresentavam parâmetros de teor de hemoglobina ( $\mathrm{g} / \mathrm{und}$ ) e hematócrito (\%) dentro do esperado para cada tipo de bolsa, conforme previsto na Portaria de Consolidação nº 05/2017 do Ministério da Saúde ${ }^{8}$.

\section{Critérios éticos}

Por se tratar de estudo retrospectivo para a obtenção de apenas dados numéricos de teor de hemoglobina ( $\mathrm{g} / \mathrm{und}$ ) e hematócrito (\%) através 
dos números de registro das bolas de concentrado de hemácia de baixo volume (CHBV) provenientes da rotina da gerência de controle de qualidade de hemocomponentes (GECOQ) da Fundação HEMOPA, sem qualquer identificação dos dados pessoais dos sujeitos da pesquisa, os pesquisadores envolvidos então assinaram Termo de Responsabilidade de Uso, Sigilo e Guarda de Dados junto à instituição promotora, situação essa que é prevista na Resolução CNS N.0 466/2012.

\section{ANÁLISE ESTATÍSTICA}

Todos os dados obtidos foram inseridos em uma planilha para posterior realização dos testes de Qui-Quadrado e determinações de médias e desvio padrão através do uso do software Graph Pad Prism 5.0, considerando-se como significativo os valores de $p \leq 0,05$.

\section{RESULTADOS}

Foi observado que $71,43 \%$ (375/525) das bolsas de CHBV analisadas, independente do tipo de bolsa, apresentavam-se em "conformidade" para os parâmetros de hematócrito e teor de hemoglobina previstos na Portaria Consolidada n ${ }^{\circ}$ 05/2017 do Ministério da Saúde ${ }^{8}$, e então, aptas para a prática transfusional (Tabela 1).

Dentre as bolsas de CHBV que se apresentavam "conformes", as bolsas duplas (Grupo I) e triplas convencionais (Grupo II) foram as que apresentaram os maiores índices de "conformidade" em relação aos parâmetros analisados e, consequentemente, o menor descarte. Por sua vez, as bolsas triplas TAB (Grupo III) e bolsas quádruplas TAB (Grupo IV) foram as que apresentaram os maiores índices de "nãoconformidade" e de descartes (Tabela 1).

Em relação aos critérios de liberação das bolsas de CHBV "conformes", foi observado que as bolsas duplas e triplas convencionais (Grupos I e II) apresentaram os maiores valores de teor de hemoglobina (Tabela 2) quando comparadas com as bolsas do tipo TAB (Grupos III e IV). Já em relação à análise de volume final das bolsas de CHBV produzidas, observou-se que as bolsas duplas (Grupo I) e as bolsas quádruplas TAB (Grupo IV) apresentaram os menores volumes.

Em relação às bolsas de CHBV "não-conformes" (Tabela 3), segundo os parâmetros hematológicos e de volume final observou-se que, independente do tipo de bolsa, o maior descarte dessas bolsas ocorreu quando as mesmas apresentavam volume $\leq 250 \mathrm{~mL}$ e concentração de hemoglobina $\leq 16 \mathrm{~g} / \mathrm{dL}$, em concomitância ou isoladamente.

Tabela 1. Análise dos critérios de "conformidade" das bolsas de CHBV produzidas na Fundação HEMOPA, no período de janeiro de 2015 a dezembro de 2018, de acordo com os parâmetros estabelecidos pela Portaria de Consolidação no 05/2017 do Ministério da Saúde ${ }^{13}$.

\begin{tabular}{lcccc}
\hline $\begin{array}{l}\text { Tipo de } \\
\text { Bolsa }\end{array}$ & $\begin{array}{c}\text { Conforme } \\
\text { \# }(\%)\end{array}$ & $\begin{array}{c}\text { Não } \\
\text { conforme } \\
\text { \# }(\%)\end{array}$ & n & p \\
\hline Grupo I & $173(89,2 \%)$ & $\begin{array}{c}21 \\
(10,8 \%)\end{array}$ & 194 & $0.0001^{*}$ \\
Grupo II & $72(65,4 \%)$ & $\begin{array}{c}38 \\
(34,6 \%)\end{array}$ & 110 & $0.0001^{*}$ \\
Grupo III & $114(58,8 \%)$ & $\begin{array}{c}80 \\
(41,2 \%)\end{array}$ & 194 & $0.0001^{*}$ \\
Grupo IV & $16(59,3 \%)$ & $\begin{array}{c}11 \\
(40,7 \%)\end{array}$ & 27 & $0.0001^{*}$ \\
TOTAL & $375(71,4 \%)$ & $\begin{array}{c}150 \\
(28,6 \%)\end{array}$ & 525 & \\
\hline
\end{tabular}

Grupo I - Concentrados de hemácias de baixo volume (CHBV) provenientes de bolsas duplas; Grupo II - CHBV provenientes de bolsas triplas convencionais; Grupo III - CHBV provenientes de bolsas triplas, modelo top and bottom (TAB); Grupo IV - CHBV provenientes de bolsas quádruplas TAB; \# ( $\%)$-valores absolutos (\#) e valores relativos (\%); $n$ - número total de amostras; $p$ - índice de significância para o Teste do Qui-Quadrado.

\section{DISCUSSÃO}

A produção de bolsas de sangue total com volume inferior ao esperado, embora não seja desejável, é prevista na Portaria de Consolidação no 05/2017 do Ministério da Saúde $^{8}$ e é considerada aceitável para bolsas de sangue total com volumes entre $300-404 \mathrm{~mL}$. Contudo, neste estudo, após a padronização in house do volume mínimo para a coleta das bolsas de sangue total entre 350 e $404 \mathrm{~mL}$, observou-se que as bolsas de CHBV geradas apresentaram os menores índices de descarte em relação aos parâmetros hematológicos estabelecidos na legislação vigente ${ }^{8}$. Quanto ao descarte das bolsas de CHBV, observou-se que o maior descarte ocorreu para bolsas com volumes finais $\leq 250 \mathrm{~mL}$ e concentração de hemoglobina $\leq 16 \mathrm{~g} / \mathrm{dL}$, em concomitância ou isoladamente. Estabelecendo-se, assim, esses critérios como maiores para o descarte de bolsas de CHBV.

As bolsas de CHBV consideradas "conformes" para os parâmetros da hematologia estabelecidos na legislação vigente ${ }^{8}$ foram submetidas as demais 
etapas do ciclo do sangue e identificadas com uma etiqueta própria de bolsa de concentrado de hemácias de baixo volume até a sua liberação para uso transfusional, conforme também é descrito em outros estudos da literatura $4,7,8,9,10,11,14,15,16,17$.

Quanto à dispensação das bolsas de CHBV "conformes", embora esse não tenha sido objeto do nosso estudo, nós entendemos que para essas bolsas deveriam existir critérios e protocolos próprios, como, por exemplo, tempos de estocagem diferenciados em relação às bolsas de $\mathrm{CH}$, com tempos menores de armazenamento para as bolsas de $\mathrm{CHBV}$, e ainda destinação específica para essas bolsas como: para reserva para cirurgias eletivas de baixo risco, para pacientes jovens sem doenças graves e para crianças maiores que quatro meses sob boas condições clínicas, estáveis hemodinamicamente, ou que precisem apenas de reserva para cirurgias não-

Tabela 2. Análise dos critérios de "conformidade" das bolsas de CHBV produzidas na Fundação HEMOPA, no período de janeiro de 2015 a dezembro de 2018, para os parâmetros hematológicos estabelecidos pela Portaria Consolidada no 05/2017 do Ministério da Saúde ${ }^{13}$

\begin{tabular}{|c|c|c|c|c|c|c|}
\hline Grupos & & $\begin{array}{c}\text { Volume final da } \\
\text { bolsa de CHBV } \\
(\mathrm{mL})\end{array}$ & $H b(g / d L)$ & Teor Hb (g/und) & Ht (\%) & $\mathbf{n}$ \\
\hline I & $X \pm$ D.P & $232,9 \pm 23,1$ & $23,5 \pm 1,1$ & $54,6 \pm 5,9$ & $72,2 \pm 2,8$ & 173 \\
\hline II & $X \pm D . P$ & $293,7 \pm 32,3$ & $17,8 \pm 1,9$ & $52 \pm 5,9$ & $54,3 \pm 4,9$ & 72 \\
\hline III & $X \pm D . P$ & $269,1 \pm 26,2$ & $18,3 \pm 1,8$ & $49 \pm 5,2$ & $55,5 \pm 5,4$ & 114 \\
\hline IV & $X \pm$ D.P & $252 \pm 22,7$ & $18,7 \pm 1,2$ & $47,1 \pm 5$ & ----- & 16 \\
\hline \multicolumn{6}{|c|}{ TOTAL } & 375 \\
\hline
\end{tabular}

Grupo I - Concentrados de hemácias de baixo volume (CHBV) provenientes de bolsas duplas; Grupo II - CHBV provenientes de bolsas triplas convencionais; Grupo III - CHBV provenientes de bolsas triplas, modelo top and bottom (TAB); Grupo IV - CHBV provenientes de bolsas quádruplas TAB; $\mathrm{n}$ - número total de amostras; $\mathrm{Hb}$ - hemoglobina, $\mathrm{Hto}$ - hematócrito; $\mathrm{X} \pm \mathrm{D} . \mathrm{P}$ - média $(\mathrm{X})$ mais ou menos desvio padrão (DP).

Tabela 3. Análise dos critérios de "não-conformidade" das bolsas de CHBV produzidas na Fundação HEMOPA, no período de janeiro de 2015 a dezembro de 2018, para os parâmetros hematológicos estabelecidos pela Portaria Consolidada no 05/2017 do Ministério da Saúde ${ }^{13}$

\begin{tabular}{|c|c|c|c|c|c|c|}
\hline Grupos & & $\begin{array}{c}\text { Volume final da } \\
\text { bolsa de CHBV } \\
(\mathrm{mL})\end{array}$ & $H b(g / d L)$ & $\begin{array}{l}\text { Teor Hb } \\
\text { (g/und) }\end{array}$ & Ht (\%) & $\mathbf{n}$ \\
\hline I & $X \pm$ D.P & $213,1 \pm 48,2$ & $21,2 \pm 2,8$ & $44,3 \pm 6,3$ & $65,6 \pm 8,3$ & 21 \\
\hline II & $X \pm$ D.P & $249,2 \pm 32,2$ & $17,7 \pm 3,4$ & $44,2 \pm 10,6$ & $54,6 \pm 10,4$ & 38 \\
\hline III & $X \pm$ D.P & $237 \pm 14,23$ & $16 \pm 0,9$ & $37,9 \pm 3,3$ & $49,1 \pm 3,1$ & 80 \\
\hline IV & $X \pm$ D.P & $207,5 \pm 18,7$ & $15,95 \pm 0,9$ & $33,24 \pm 3,9$ & ----- & 11 \\
\hline \multicolumn{6}{|c|}{ TOTAL } & 150 \\
\hline
\end{tabular}

Grupo I - Concentrados de hemácias de baixo volume (CHBV) provenientes de bolsas duplas; Grupo II - CHBV provenientes de bolsas triplas convencionais; Grupo III - CHBV provenientes de bolsas triplas, modelo top and bottom (TAB); Grupo IV - CHBV provenientes de bolsas quádruplas TAB; $n$ - número total de amostras; $\mathrm{Hb}$ - hemoglobina, Hto - hematócrito; $X \pm D . P$ - média mais ou menos desvio padrão.

cardíacas, visto que para esse público pediátrico, especificamente, são utilizadas apenas alíquotas de concentrados de hemácias (5-15 ml/kg) ${ }^{10,11,15,16,17}$.

Essa hipótese levantada por nós, quanto ao menor tempo de estocagem e destinação específica dessas bolsas, baseia-se no fato de que as bolsas de CHBV "conformes" para os parâmetros da hematologia estabelecidos na legislação vigente ${ }^{8}$, são derivadas de bolsas de sangue total que passaram por algum tipo de intercorrência durante a coleta e, como tal, não sabemos exatamente quais produtos biológicos (citocinas pró-inflamatórias e/ou proteínas de estresse) podem perdurar nessas bolsas e interferir na condição de saúde de um receptor já debilitado ${ }^{18}$. E ainda, porque o menor tempo de estocagem das bolsas de CHBV determinaria também o menor risco de lesões de estocagem em hemácias $3,8,9,11,18,19,20,21,22,23$ ou ainda menor influência da toxicidade do plastificante das bolsas Di(2-etilhexil ftalato) (DEHP) ao paciente $18,19,22,23$. 
Barshtein, Arbell and Yedgar ${ }^{18}$ em seus estudos quanto ao tempo de estocagem das bolsas de $\mathrm{CH}$, apontam que muitas vezes a decisão pela dispensação dessas é baseada em critérios como o tempo de estocagem da mesma, isto é, a bolsa de $\mathrm{CH}$ que primeiro foi produzida e estocada será também a primeira a ser liberada para transfusão. No entanto, segundo esses autores, a funcionalidade das hemácias transfundidas, ou seja, a sua capacidade de aumentar a hemoglobina e oxigenação dos tecidos do paciente transfundido, muitas vezes é ignorada, de modo que, por vezes o produto transfundido possui apenas a propriedade de melhorar o fluxo sanguíneo do receptor. Essas observações corroboram com a nossa hipótese da necessidade de melhor definição do tempo de estocagem também para as bolsas de CHBV.

Outra questão que não foi abordada em nosso estudo, mas que merece uma reflexão e futuros estudos para comprovação, refere-se ao possível aproveitamento das bolsas de CHBV "não-conformes" que são descartadas. Nós acreditamos, mesmo ainda de forma hipotética, que essas bolsas quando apresentam teor de hemoglobinas e hematócrito dentro dos parâmetros previstos na legislação brasileira ${ }^{8}$, independente do volume final da bolsa e ainda quando provenientes de doadores de sangue de tipos sanguíneos raros, poderiam ser utilizadas sob condições muito específicas em transfusões sanguíneas, como, por exemplo, em transfusões pediátricas para crianças maiores de quatro meses, com hemoglobina entre $6-10 \mathrm{~g} / \mathrm{dL}$, sob boas condições clínicas, estáveis hemodinamicamente, ou que precisem apenas de reserva de concentrado de hemácias para cirurgias não-cardíacas, visto que nestas condições também são utilizadas apenas alíquotas de concentrados de hemácias de menores volumes $(5-15 \mathrm{ml} / \mathrm{kg})^{7,10,11,15,16,17,19}$.

Assim, neste estudo nós consideramos importante que se inicie uma discussão mais ampla sobre o descarte de bolsas de CHBV "não-conformes" e também uma reavaliação dos parâmetros da legislação vigente quanto ao volume, o tempo de estocagem e os critérios de dispensação para as bolsas de CHBV "conformes".

\section{CONCLUSÃO}

Neste estudo observou-se que o melhor aproveitamento das bolsas de concentrados de hemácias de baixo volume produzidas na Fundação HEMOPA ocorreu quando as bolsas de sangue total se encontravam com volumes entre 350 e $404 \mathrm{~mL}$ e as bolsas de CHBV apresentavam volume final $\geq$ $250 \mathrm{~mL}$ e hemoglobina $\geq 16 \mathrm{~g} / \mathrm{dL}$.

\section{BIBLIOGRAFIA}

1. Rodrigues RSM, Reibnitz KS. Estratégias de Captação de Doadores de Sangue: Uma revisão integrativa da literatura. Texto Contexto Enferm. 2011;20(2):384-91.

2. Zago A, Da Silveira MF, Dumith SC. Prevalência de doação de sangue e fatores associados, Pelotas, RS. Rev. Saúde Pública. 2010;44(1):112-20.

3. Kitpoka P, Chanthet S, Chongkolwatana V, Chuansumrit A, Kunakorn M. Comparison of Double RBC Collection by Blood Cell Separators. J Med Assoc Thai. 2016;99(1):88-96.

4. Barbosa SM, Torres CA, Gubert FA, Pinheiro PNC, Vieira NFC. Enfermagem e a prática hemoterápica no Brasil: revisão integrativa. Acta Paul. Enferm. 2011;24(1):132-6.

5. Martins PRJ, Martins RA, Barbosa VF, Pereira GA, MoraesSouza $H$, Silva SS. The importance of hemovigilance in the transmission of infectious diseases. Rev. Bras. Hematol. Hemoter. 2013;35(3):180-184. doi: 10.5581/1516-8484.20130040.

6. Bihl F, Castelli D, Marincola F, Dodd RY, Brander C. Transfusion-transmitted infections. J Transl Med. 2007;6(5):25. doi: 10.1186/1479-5876-5-25

7. Bittencourt R, Costa J, Lobo JEO, Aguiar FC. Transfusão Consciente de Hemoderivados. Revisão Sistemática dos Fatores Indicativos do Gatilho para a Infusão dos Componentes Sanguíneos. Revista Brasileira de Anestesiologia. 2012;62(3):402-10.

8. Brasil. Ministério da Saúde. Portaria de Consolidação nº 05 de 28/07/2017, do Ministério da Saúde. Brasília: Diário Oficial da União, 2017.

9. Czaikoski PG, Moreira RC, Ferreira H, Monteiro MC. Controle de Qualidade em Concentrado de Hemácia no Hemonúcleo de Guarapuava - PR. Rev. Salus. 2008;2(2):63-72.

10. Brasil, Ministério da Saúde, Secretaria de Atenção à Saúde, Deparatamento de Atenção Especializada e Temática. Guia para uso de hemocomponentes, 2. ed., 3. Reimp. - Brasília: 136p. 2016.

11. CARSON JL, Grossman BJ, Kleinman S. Red blood cell transfusion: a clinical practice guideline from the AABB. Ann Intern Med. 2012;157(1):49-58.

12. Covo MZ, Cruz EDA, Maurício AB, Batista J, Souza LAL. Custo financeiro dos descartes de sangue total e hemocomponentes em um hemocentro coordenador brasileiro. Rev Gaúcha Enferm. 2019;40:e20190033. doi: https://doi.org/10.1590/1983-1447.2019.20190033.

13. Estácio AG. Relação de custo versus benefício do descarte de concentrado de hemácias de baixo volume na Fundação HEMOPA. [Trabalho de Conclusão de Curso da Especialização em Gestão de Hemocentro (GHEMO)]. Rio de Janeiro: Escola Nacional de Saúde Pública Sérgio Arouca - Fundação Oswaldo Cruz, FIOCRUZ, Brasil; 2017.

14. Corwin HL, Gettinger A, Pearl RG, Fink MP, Levy MM, Abraham E, MacIntyre NR, ShabotMM, Duh MS, Shapiro MJ. The Critical Study: Anemia and blood transfusion in the critically ill-current clinical practice in the United States. Crit. Care Med. 2004;32(1):39-52. 
15. Campos LR, Cerqueira AJB, Campos CJB, Pereira de Souza JGB, Novello R, Rosa Pessôa VL, Feitosa ACF. Transfusão de hemocomponentes em crianças: o quê, quando e como usar?. Residência Pediátrica. 2015;5(1):14-20.

16. Laverdière $C$, Gauvin F, Hébert PC, Infante-Rivard $C$, Hume $H$, Toledano BJ, et al. Survey on transfusion practices of pediatric intensivists. Pediatr Crit Care Med. 2002;3(4):335-40.

17. Mendes C, Bourguignon da Silva DC, Arduini RG, Troster EJ. Prática de transfusão de concentrado de hemácias sem Unidade de Terapia Intensiva Pediátrica. Einstein. 2011;9(2 Pt 1):135-9.

18. Barshtein G, Arbell D, Yedgar S. Hemodynamic Functionality of Transfused Red Blood Cells in the Microcirculation of Blood Recipients. Front. Physiol. 2018;9(41):1-6. doi: 10.3389/fphys.2018.00041.

19. Tzounakas VL, Valsamib SI, Kriebardisc AG, Papassideria IS, Seghatchian DJ, Antonelou MH. Red cell transfusion in paediatric patients with thalassaemia and sickle cell disease: Current status, challenges and perspectives. Transfusion and Apheresis Science. 2018;57:347-357.
20. Rapido F, Brittenham GM, Bandyopadhyay S, La Carpia F, L'Acqua C, McMahonDJ, et al. Prolonged red cell storage before transfusion increases extravascularhemolysis. J Clin Invest 2017;127(1):375-82.

21. Kalhan TG, Bateman DA, Bowker RM, Hod EA, Kashyap S. Effect of red blood cellstorage time on markers of hemolysis and inflammation in transfused very lowbirth weight infants. Pediatr Res 2017;82(6):964-9.

22. Fields $M E$, Hulbert $M L$, Chen $L$, Berlin $A N$, Jackups $R$, Spinella PC. Red blood cellstorage duration is not associated with clinical outcomes for acute chest syndromein children with sickle cell disease. Transfusion 2015;55(11):2714-21.

23. Dhabangi A, Ainomugisha B, Cserti-Gazdewich C, Ddungu $H$, Kyeyune D, Musisi E, et al. Effect of transfusion of Red blood cells with longer vs shorter storageduration on elevated blood lactate levels in children with severe Anemia: the TOTAL randomized clinical trial. JAMA 2015;314(23):2514-23 


\section{Agradecimentos}

A Fundação HEMOPA pela liberação da autora para a realização do mestrado e a equipe do Laboratório de Controle de Qualidade do HEMOPA - Ana Carolina Neves, Roberta Nice Sodré, Thais de Figueiredo, Ruth Souza e Waldecir Duarte pelo empenho e profissionalismo no auxílio deste estudo.

\section{Conflito de interesses}

Não há.

Financiamento da pesquisa

Universidade Federal do Pará.

Instituições onde foi realizado o estudo'

Trabalho desenvolvido no Laboratório de Patologia Geral - Imunopatologia e Citologia da UFPA em parceria com a Fundação HEMOPA.

\section{Autor Correspondente:}

Prof. Dr. Lacy Cardoso de Brito Junior

Icdbrito@ufpa.br, Icdbrito2@gmail.com

Editor:

Prof. Dr. Marcelo Riberto

Recebido: 05/05/2021

Aprovado: 18/06/2021 